\title{
Correction: MicroRNA-27a promotes podocyte injury via PPARY-mediated $\beta$-catenin activation in diabetic nephropathy
}

Zhanmei Zhou', Jiao Wan', Xiaoyan Hou ${ }^{1,2}$, Jian Geng ${ }^{3}$, Xiao Li ${ }^{4}$ and Xiaoyan Bai ${ }^{1}$

Correction to: Cell Death and Disease 8, e2658 (2017); https://doi.org/10.1038/cddis.2017.74; published online 9 March 2017

Since the publication of this article, the authors identified an omission in the funding information. The original funding information was as follows:

This study was supported by National Nature and Science Young Investigator Grant (no. 81100496) from the National Natural Science Foundation of China, Special Fund from Chinese Society of Nephrology (no. 13030370422), Guangdong Natural Science Foundation (no. 2016A030313581) and Distinguished Young Scholar Fund from Nanfang Hospital (no. 2015J009) to X.B. We thank Guangzhou King Medical Diagnostics Center for providing human renal biopsy samples. We gratefully acknowledge all lab members for their technical assistance.

The authors would also like to acknowledge the following:

Guangzhou Science and Technology Planning ProjectKey Projects of Scientific Research (201607020019) and Distinguished Young Scholar Fund from Nanfang Hospital (no. 2015J009) to X.B.

The authors apologise for any inconvenience caused. 